\title{
Perceived versus proven SARS-CoV-2-specific immune responses in health-care professionals
}

\author{
Georg M. N. Behrens ${ }^{1,2} \cdot$ Anne Cossmann ${ }^{1,2} \cdot$ Metodi V. Stankov ${ }^{1,2} \cdot$ Torsten Witte $^{1} \cdot$ Diana Ernst $^{1}$. \\ Christine Happle ${ }^{3,4} \cdot$ Alexandra Jablonka $^{1,2}$
}

Received: 12 May 2020 / Accepted: 31 May 2020 / Published online: 10 June 2020

(c) The Author(s) 2020

\begin{abstract}
There have been concerns about high rates of thus far undiagnosed SARS-CoV-2 infections in the health-care system. The COVID-19 Contact (CoCo) Study follows 217 frontline health-care professionals at a university hospital with weekly SARSCoV-2-specific serology (IgA/IgG). Study participants estimated their personal likelihood of having had a SARS-CoV-2 infection with a mean of $21 \%$ [median $15 \%$, interquartile range (IQR) 5-30\%]. In contrast, anti-SARS-CoV-2 IgG prevalence was about $1-2 \%$ at baseline. Regular anti-SARS-CoV-2 IgG testing of health-care professionals may aid in directing resources for protective measures and care of COVID-19 patients in the long run.
\end{abstract}

Keywords COVID-19 $\cdot$ SARS-CoV-2 $\cdot$ Immunoglobulin $\cdot \operatorname{IgG} \cdot \operatorname{IgA} \cdot$ Health-care worker $\cdot$ ELISA $\cdot$ Seroprevalence Diagnostics $\cdot$ Health-care professionals

\section{Abbreviations \\ COVID-19 Coronavirus disease 19 \\ ELISA Enzyme-linked immunosorbent assay \\ IgG Immunoglobulin $\mathrm{G}$ \\ PCR Real-time polymerase chain reaction}

SARS-CoV-2 Severe acute respiratory syndrome coronavirus 2

Despite growing access of broadly available testing systems, uncertain rates of asymptomatic infections have raised

Christine Happle and Alexandra Jablonka contributed equally to this work.

Georg M. N. Behrens

behrens.georg@mh-hannover.de

1 Department for Rheumatology and Clinical Immunology, Hannover Medical School, Carl-Neuberg-Straße 1, 30625 Hannover, Germany

2 German Center for Infection Research (DZIF), partner site Hannover-Braunschweig, Hannover, Germany

3 Department of Pediatric Pneumology, Allergology, and Neonatology, Hannover Medical School, Hannover, Germany

4 German Center for Lung Research, Biomedical Research in End Stage and Obstructive Lung Disease/BREATH Hannover, Hannover, Germany concerns about a potentially high rate of thus far undiagnosed SARS-CoV-2 infections, particularly in frontline medical staff [1]. To prevent the breakdown of health-care systems during the current pandemic, the protection of medical personnel and patients from contracting a SARS-CoV-2 infection is central [2].

Consent finding for case definition, COVID-19 diagnosis in suspected cases, and scaling up of suitable diagnostic systems have been challenging since the start of the pandemic. Real-time polymerase chain reaction (PCR)-based nasopharyngeal (or throat) swab testing was rapidly developed and has helped in ascertainment and tracking of the SARSCoV-2 outbreak [2]. However, the sensitivity of PCR-based testing, which is thus far only applied routinely for symptomatic patients, crucially depends on the timing and type of respiratory sampling and led to false negative rates of up to $70 \%$ during the early phase of the pandemic [3, 4]. Serological testing for SARS-CoV-2-specific immunoglobulins (Ig) is relatively easy, inexpensive, and critical for epidemiological studies. SARS-CoV-2-specific B cell responses appear to correlate to disease severity with rising antibody titers typically between 5 to 10 days and fully positive rates at about 18 days after symptom onset [5]. As such, serological testing can be helpful in suspected cases with negative PCR results and in identification of asymptomatic infections [6].

We initiated the COVID-19 Contact (CoCo) study to weekly monitor SARS-CoV-2-specific serology (IgA/IgG) 
in frontline health-care professionals (HCP) in combination with a questionnaire about respiratory symptoms and risk perception. As testing system, we employed a semiquantitative ELISA [EUROIMMUN Medizinische Labordiagnostik, Lübeck, Germany-CE certified version: specificity $99.0 \%$, sensitivity $93.8 \%$ after day 20 according to the manufacturer [5]]. We confirmed the specificity in a set of 156 sera from non-European refugees and migrants [7] collected in 2015 as negative controls (mean age 31.6 years, range 18-67 years, $78 \%$ male). All but one tested negative for SARS-CoV-2 IgG (specificity $99.3 \%$ ) and 2 out of 90 tested equivocal positive for IgA (specificity 97.8\%). The serum of 18 patients after recovery of PCR-confirmed COVID-19 served as positive controls or to generate a standard curve (mean age 44.8 years; mean duration of symptoms 11.8 days, range 3-35 days; mean time since start of symptoms 30.4 days, range $21-61$ days). $16 / 18$ tested positive ( $n=1$ equivocal positive) for SARS-CoV-2 IgG (sensitivity $90 \%$ ) and $15 / 18$ positive for SARS-CoV-2 IgA (sensitivity $85.7 \%$ ). Interestingly, the duration of symptoms as a surrogate for disease severity correlated significantly with the IgG ratio (extinction of sample to calibrator ratio) of the SARS-CoV-2 IgG ELISA (Fig. 1a).

Between March 23 and April 17, $n=217$ HCP from emergency rooms, infectious and pulmonary disease wards, ICUs, pediatric departments and other units involved in COVID-19 patient care at our university hospital were included in the study. The mean age of participants was 36.5 years (range 18-63 years), and 65\% were female. Most of them worked as physicians (53.5\%), nurses (27.6\%), or medical assistants $(9.2 \%)$. The majority of participants had direct contact with patients with infectious respiratory diseases working in the emergency department (40.1\%), general ward (31.8\%), or outpatient departments (13.8\%). At baseline, $1.6 \%$ of included personnel reported to have visited regions with high SARS-CoV-2 prevalence as defined by the German National Institute of Public Health (Robert Koch Institute
[8]), $16.1 \%$ reported to have had contact with confirmed COVID-19 cases, and more than one-third (39.2\%) to have had contact with suspected COVID-19 cases. $45.2 \%$ of HCP reported to suffer from at least one respiratory symptom of any severity, and $29.0 \%$ reported to have had a respiratory infection during the past 2 weeks.

Upon enrollment, study participants were asked to estimate their personal likelihood of having had a SARS-CoV-2 infection (How high do you rate the probability of having been infected so far? $0-100 \%$ ). Only $12 \%$ of the $n=201$ study participants, who answered this question, rated a $0 \%$ chance of having already had contracted SARS-CoV-2, while $19 \%$ rated their probability greater than $50 \%$. Strikingly, the mean percentage of self-perceived positive SARSCoV-2 infection status was $21 \%$ (range $0-90 \%$, median $15 \%$, IQR 5-30\%). Male participants rated their infection risk lower than female participants (mean $16.2 \%$ vs. $23.7 \%$, $F=4.4, p=0.02$, median $10 \%$ vs. $20 \%, Z=3.4, p=0.001$ ) and older subjects reported lower infection probabilities as compared to younger participants (Pearson -0.33 , $p=0.004$ ) (Fig. 1b). Reported contact to confirmed or suspected COVID-19 cases did not have a significant impact on perceived probability of infection.

In contrast to the high percentage of self-perceived positive SARS-CoV-2 infection status, only two of $n=217$ tested frontline HCP showed a clearly positive reaction in the ELISA, and two displayed equivocal positive results according to the manufacturer's interpretation. Both positive results were about 20 -fold lower as compared to one patient with severe COVID-19 (Fig. 1a). The majority of participants $(n=214)$ had no evidence of anti-SARS-CoV-2 IgG. Anti-SARS-CoV-2 IgA was positive/equivocal positive in $n=9$ and $n=10$ subjects, respectively, and combined $\operatorname{IgG}$ and IgA-positive/equivocal positive in three subjects. Altogether, anti-SARS-CoV-2 IgG prevalence was in the range of $1-2 \%$ and the self-perceived likelihood of SARS-CoV-2 infection in these individuals similar to the entire cohort.

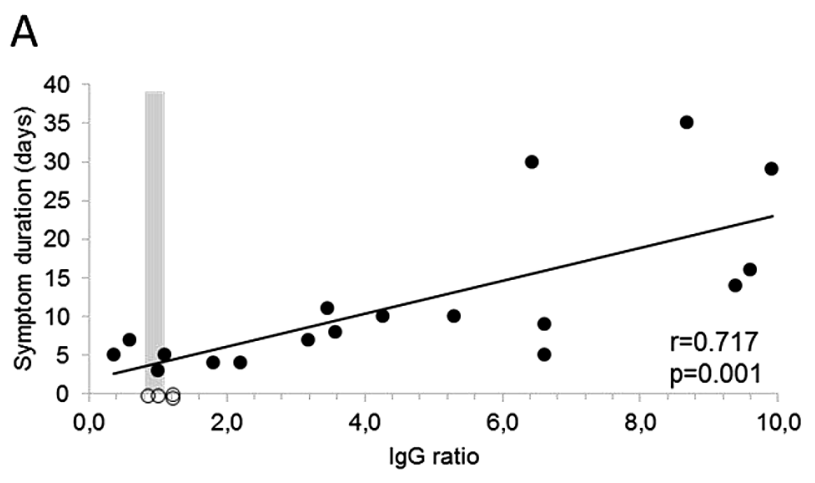

Fig. 1 a Anti-SARS-CoV-2 IgG ELISA results. PCR-confirmed COVID-19 cases are depicted as black dots, and health-care professionals depicted as open dots (for which symptoms were not consid-

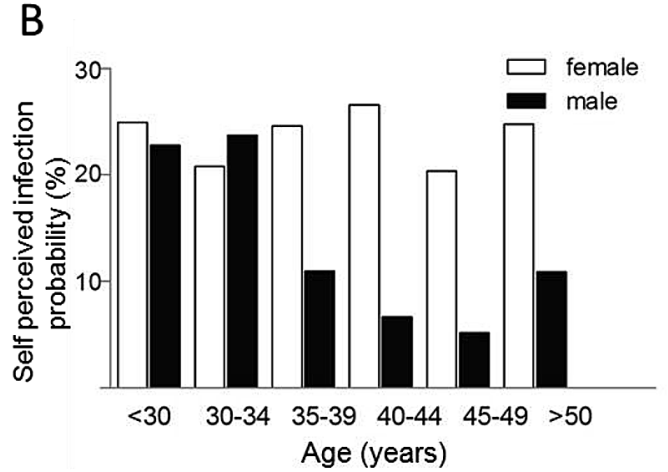

ered). The gray zone (0.8-1.1 ratio) represents the range with equivocal ELISA results. b Differences in mean self-perceived probability for SARS-CoV-2 infection in relation to sex and age 
Our data on SARS-CoV-2 IgG is only partially representative for our university hospital, not fully representative for other clinics, and we do not know the source of infection in anti-SARS-CoV-2 IgG-positive HCP. However, the gap between perceived risk and evidence for an infection is most likely a phenomenon in many health-care settings. Additionally, we have only limited information about the full validity of anti-SARS-CoV-2 serology tests for screening. In a setting with low COVID-19 prevalence, the use of the spike protein $\mathrm{S} 1$ to screen for anti-SARS-CoV-2 IgG may be suboptimal, and testing for antibodies against, e.g., the receptor-binding domain of SARS-CoV-2 could increase sensitivity. Interestingly, given the significant association between disease severity and anti-SARS-CoV-2 IgG in our ELISA, we hypothesize that asymptomatic seroconversions could lead to numerous equivocal positive ELISA results (Fig. 1a), which still may represent neutralizing activity [9]. Such data may be difficult to interpret in cross-sectional studies and our longitudinal study design combined with neutralization assays will be informative about the magnitude of ELISA result changes over time. Finally, a matter of debate remains whether serological tests can also inform about COVID-19-specific immunity. Preliminary studies in rhesus macaques suggest that reinfection does not occur after survival of COVID-19, supporting the notion of at least temporary immunity after primary infection [10].

Taking these limitations into account, our data point toward a currently low rate of SARS-CoV-2-specific IgG in HCP in Northern Germany hospitals, where no overflow of COVID-19 patients has challenged the health-care system so far and confirmed outbreaks are limited. This is in stark contrast to the relatively high rate of self-estimated SARS-CoV-2 infection probability of our hospital's frontline HCP and strikingly different from currently high infection rates in medical personnel from Italian regions [1]. Also, our data show that personal risk perception correlates to age and sex, which should be taken into account when advising hospital staff on protective measures against COVID-19. Regular anti-SARS-CoV-2 IgG testing of health-care workers may aid in monitoring the pandemic, assessing the quality of immune responses, and directing resources to assure COVID-19 care in the long run.

Acknowledgements Open Access funding provided by Projekt DEAL. We thank the study site coordinators and participants of the $\mathrm{CoCo}$ Study group.

Author contributions $\mathrm{GB}, \mathrm{CH}$, and $\mathrm{AJ}$ designed and managed the project, analyzed the data and drafted the manuscript. AC managed proband inclusion, designed the workflow and questionnaires, and organized sample collection. MS performed the experiments and designed the laboratory workflow, DE and TW were involved in planning and supervision of the project. All authors discussed the results and approved the final manuscript.
Funding There has been no external funding for this project.

\section{Compliance with ethical standards}

Conflict of interest AJ reports grants and personal fees from Novartis, grants and personal fees from Abbvie, grants and personal fees from Gilead, and personal fees from Roche outside the submitted work. TW reports grants and personal fees from Novartis, grants and personal fees from Abbvie, personal fees from Gilead, personal fees from Chugai, personal fees from Sanofi-Aventis, and non-financial support from Aesku Diagnostics, outside the submitted work. DE reports grants and personal fees from Novartis, grants and personal fees from Abbvie, grants and personal fees from Gilead, personal fees from Sanofi Aventis, and personal fees from GSK, outside the submitted work. GB reports grants and personal fees from Gilead, and personal fees from ViiV Healthcare, MSD, and Janssen outside the submitted work. Other authors have nothing to disclose.

Ethics approval The here presented analyses were approved by local authorities (Data Security Management and Institutional Review Board of Hannover Medical School, approval number 8973_BO_K_2020). Informed consent was obtained from all participants.

Availability of data and materials The datasets used and/or analyzed during the current study are available from the corresponding author on reasonable request.

Open Access This article is licensed under a Creative Commons Attribution 4.0 International License, which permits use, sharing, adaptation, distribution and reproduction in any medium or format, as long as you give appropriate credit to the original author(s) and the source, provide a link to the Creative Commons licence, and indicate if changes were made. The images or other third party material in this article are included in the article's Creative Commons licence, unless indicated otherwise in a credit line to the material. If material is not included in the article's Creative Commons licence and your intended use is not permitted by statutory regulation or exceeds the permitted use, you will need to obtain permission directly from the copyright holder. To view a copy of this licence, visit http://creativecommons.org/licenses/by/4.0/.

\section{References}

1. Rosenbaum L. Facing Covid-19 in Italy-ethics, logistics, and therapeutics on the epidemic's front line. N Engl J Med. 2020. https://doi.org/10.1056/NEJMp2005492.

2. Ferioli M, Cisternino C, Leo V, Pisani L, Palange P, Nava S. Protecting healthcare workers from SARS-CoV-2 infection: practical indications. Eur Respir Rev. 2020. https://doi.org/10.1183/16000 617.0068-2020.

3. Yang Yang MY, Chenguang S, Fuxiang W, Jing Y, Jinxiu L, Mingxia Z, Zhaoqin W, Li X, Jinli W, Ling P, Gary W, Haixia Z, Mingfeng L, Kai F, Jianming L, Qianting Y, Juanjuan Z, Zheng Z, Lei L, Yingxia L. Evaluating the accuracy of different respiratory specimens in the laboratory diagnosis and monitoring the viral shedding of 2019-nCoV infections. medrxiv. 2020. https://doi. org/10.1101/2020.02.11.20021493v2.

4. Ai T, Yang Z, Hou H, Zhan C, Chen C, Lv W, Tao Q, Sun Z, $\mathrm{Xia}$ L. Correlation of chest CT and RT-PCR testing in coronavirus disease 2019 (COVID-19) in China: a Report of 1014 Cases. Radiology. 2020:200642. 
5. Okba NMA, et al. SARS-CoV-2 specific antibody responses in COVID-19 patients. Emerg Infect Dis. 2020. https://doi. org/10.3201/eid2607.200841.

6. Long Q, Liu B, Deng H, et al. Antibody responses to SARSCoV-2 in patients with COVID-19. Nat Med. 2020. https://doi. org/10.1038/s41591-020-0897-1.

7. Jablonka A, et al. Seroprevalence of antibodies and antigens against hepatitis A-E viruses in refugees and asylum seekers in Germany in 2015. Eur J Gastroenterol Hepatol. 2017;29:939-45.

8. Robert Koch Institute. Coronavirus Disease 2019 (COVID-19) Daily situation report of the Robert Koch Institute. https://www. rki.de/DE/Content/InfAZ/N/Neuartiges_Coronavirus/Situations berichte/2020-03-23-en.pdf. Access: 05/03/2020

9. Streeck H, Schulte B, Kümmerer BM, Richter E, Höller T, Fuhrmann C, Bartok E, Dolscheid R, Berger M, Wessendorf
L, Eschbach-Bludau M, Kellings A, Schwaiger A, Coenen M, Hoffmann P, Stoffel-Wagner B, Nöthen MM, Eis-Hübinger AM, Exner M, Schmithausen RM, Schmid M, Hartmann G. Infection fatality rate of SARS-CoV-2 infection in a German community with a super-spreading event. medrxiv. 2020. https://doi. org/10.1101/2020.05.04.20090076.

10. Linlin Bao WD, Hong G, Chong X, Jiayi L, Jing X, Qi L, Jiangning L, Pin Y, Yanfeng X, Feifei Q, Yajin Q, Fengdi L, Zhiguang X, Haisheng Y, Shuran G, Mingya L, Guanpeng W, Shunyi W, Zhiqi S, Wenjie Z, Yunlin H, Linna Z, Xing L, Qiang W, Chuan Q. Lack of reinfection in rhesus macaques infected with SARSCoV-2. biorxiv. 2020. https://doi.org/10.1101/2020.03.13.99022 6. 